\title{
Sistema de calificación para juegos motores en función de la edad
}

\section{Rating system for motor games based on age}

\author{
Andrés B. Fernández-Revelles ${ }^{1 *}$, Ruben Moreno-Arrebola ${ }^{2}$, Tamara Espejo-Garcés ${ }^{3}$, Federico Báez-Mirón ${ }^{4}$ \\ e Irwin A. Ramírez-Granizo
}

\begin{abstract}
1 * Departamento de Educación Física y Deportiva, Facultad de Ciencias del Deporte, Universidad de Granada (España). ORCID https://orcid.org/0000-00028089-650X

2 Grupo de Investigación HUM-386 (FORCE) Facultad de Ciencias de la Educación, Universidad de Granada (España). ORCID http://orcid.org/0000-00021270-393X

3 Departamento de Didáctica. Facultad de Ciencias de la Educación. Universidad de Cádiz (España). ORCID https://orcid.org/0000-0003-0363-7464

4 Departamento de Didáctica de la Expresión Musical, Plástica y Corporal, Universidad de Jaén (España).

5 Departamento de Didáctica de la Expresión Musical, Plástica y Corporal, Universidad de Granada (España). ORCID https://orcid.org/0000-0002-6944-
\end{abstract} 3737

Resumen: Introducción: Partiendo de la necesidad de calificación de los juegos motores para orientar a los menores y adolescentes en su uso. Objetivo: Proponer las bases para un sistema de calificación, evaluación y clasificación de juegos motores partiendo de los sistemas de calificación de videojuegos. Metodología: Análisis de los sistemas de calificación de videojuegos. Resultados y Discusión: Se han encontrado diferentes sistemas de calificación por edades de videojuegos basadas en los descriptores: violencia, sexo y adicciones que modifican su impacto en función de diferentes moduladores. Tienen bastantes similitudes y están extendidos por todo el mundo. Conclusiones: Se pueden establecer las bases para una propuesta de un sistema de calificación de juegos motores partiendo de los sistemas de calificación de videojuegos, modificando calificación de edad, descriptores y moduladores en relación con la actividad motora.

Palabras clave: Sistemas de calificación de juegos; videojuegos, juegos motores, protección de menores.
Abstract: Introduction: Starting from the need to qualify motor games to guide children and adolescents in its use. Aim: Propose the bases for a motor games rating system starting from the videogame rating system. Methodology: Analysis of videogame rating systems. Results and Discussion: We have found different age rating systems for video games based on the descriptors: violence, sex and addictions that modify their impact depending on different modulators. They have many similarities and are widespread throughout the world. Conclusions: The bases for a proposal of a motor games rating system can be established starting from the systems of qualification of videogames, modifying qualification of age, descriptors and modulators in relation to the motor activity.

Keywords: Game rating systems; video games, motor games, child protection.

\section{Introducción}

En la sociedad que vivimos hoy día los niños y adolescentes pasan numerosas horas al día, a la semana, al mes una gran parte de su tiempo de ocio jugando a videojuegos (Anders, 1999).

Este comportamiento generalizado en los menores de edad de todo el mundo repercute en una disminución del tiempo dedicado a actividad física y a estar influenciados por todo el entorno digital y fantástico que viven a través de los videojuegos. Pero no todos los videojuegos son iguales ni están realizados para que se juegue a ellos a cualquier edad (Jordan, 2008).

Así a mediados de los años 90 en el siglo XX surge la necesidad de crear sistemas de calificación, evaluación y clasificación de los videojuegos (Funk, Flores, Buchman, \& Germann, 1999). Parten de la idea ya existente de calificación

Dirección para correspondencia [Correspodence address]: Andrés B. Fernández-Revelles Departamento de Educación Física y Deportiva, Facultad de Ciencias del Deporte, Universidad de Granada (España). E-mail: abfr@ugr.es, ORCID https://orcid.org/0000-0002-8089-650X de otros productos de entretenimiento como DVD, vídeos, películas etc... (Gentile, Maier, Hasson, \& de Bonetti, 2011; Walsh \& Gentile, 2001).

Los videojuegos forman parte del entorno de enseñanzaaprendizaje en el que vive el niño (Sedeno, 2010), por tanto es muy importante orientar a los menores hacia los videojuegos más adecuados para su edad (Jordan, 2008).

La calificación de los videojuegos para tener una orientación adecuada en su uso viene determinada por diferentes descriptores como son contenidos de violencia (Becker-Olsen \& Norberg, 2010; Bijvank, Konijn, Bushman, \& Roelofsma, 2009; Funk et al., 1999; Laczniak, Carlson, Walker, \& Brocato, 2017), el consumo de drogas y de alcohol (Cranwell, Whittamore, Britton, \& Leonardi-Bee, 2016), utilización de lenguaje soez, contenidos con juegos de azar y apuestas, desnudos y contenido sexual, escenas que provocan miedo etc... (Dogruel \& Joeckel, 2013).

Debido a la necesidad de calificación de los videojuegos se han creado en todo el mundo diferentes sistemas de calificación de videojuegos o en, terminología inglesa Game Rat- 
ing Systems, siendo los sistemas de calificación de videojuegos más extendidos EntertainmentSooftware Rating Board (ESRB) que es utilizado en Norteamérica (Entertainment Software Association, 2017) y el más extendido en Europa es Pan EuropeanGameInformation (PEGI) (Pan European Game Information (PEGI), 2017). Incluso ha aparecido en 2013 una agencia con el fin de unificar los diferentes sistemas de calificación de los videojuegos, es la International Age Rating Coalition (IARC) (International Age Rating Coalition (IARC), 2017) cuya finalidad es simplificar el proceso de calificación de los videojuegos y adaptarlo a los sistemas de evaluación regionales tal y como indican (Fernández-Revelles, 2017g).

Nos hemos centrado en los sistemas de calificación de los videojuegos porque al ser unos sistemas extendidos y conocidos por los niños de todo el mundo va a ser más fácil realizar una adaptación de estos sistemas al entorno de los juegos motores que crear uno nuevo de la nada, también con el fin de que su familiarización sea rápida y sencilla. Además, hoy día los niños buscan los juegos motores con los que jugar a través de las redes sociales e internet (Fernández-Revelles, 2017a, 2017b, 2017c, 2017d, 2017e, 2017f).

Creemos que al igual que existe la necesidad de calificación de los videojuegos igualmente existe la necesidad de orientar en el uso de los juegos motores para orientar a los menores en un adecuado uso y para protegerlos del uso inadecuado teniendo en cuenta los mismos descriptores que utilizan los sistemas de calificación de videojuegos y además los propios de los juegos motores como la intensidad del juego, o la utilización de habilidades motrices y cualidades físicas, etc... tal y como se puede apreciar en (Fernández-Revelles, 2017b).

\section{Objetivo}

Proponer un sistema de calificación, evaluación y clasificación de juegos motores partiendo de los sistemas de calificación de videojuegos.

\section{Material y método}

\section{Análisis de los sistemas de calificación de videojuegos}

Para realizar este estudio vamos utilizar una estrategia en tres partes, empezamos en primer lugar con una revisión sistemática de las bases de datos Web ofScience (WoS) y Scopus, utilizando las palabras claves "Game Rating System" siguiendo la metodología descrita en (Fernández-Revelles, 2012, 2014; Fernández-Revelles, Chillon, et al., 2009; FernándezRevelles, Robles, et al., 2009). Se realizó la búsqueda el 30 de septiembre de 2017.
En segundo lugar, revisamos los abstracts de las publicaciones encontradas para ratificar que no existe ningún sistema de calificación de juegos motores.

En tercer lugar, realizamos una búsqueda heurística a través del buscador Google utilizando las mismas palabras claves, unida a la revisión de las publicaciones encontradas en la búsqueda en las bases de datos en las que se encuentran "Game Rating System" y examinando los sistemas de calificación de videojuegos conocidos como son IARC, ESRB y PEGI. Este trabajo se estuvo desarrollando entre del 1 al 15 de octubre de 2017.

Para realizar esta búsqueda heurística dentro de las publicaciones realizamos el siguiente algoritmo: búsqueda en las bases de datos, confección de lista de seleccionados, descarga de las referencias de las publicaciones en el gestor bibliográfico Endnote X7, buscar y adjuntar texto completo a las referencias, búsqueda con las iniciales de los sistemas de calificación encontrados en todas las publicaciones utilizando el buscador del gestor, creación de carpetas y selección de publicaciones, búsqueda de las páginas web de los distintos sistemas de calificación, recoger los sistemas de calificación.

\section{Resultados}

Los sistemas de calificación de videojuegos encontrados ha sido los siguientes:

- Pan EuropeanGameInformation (PEGI) utilizado en Europa (Pan European Game Information (PEGI), 2017).

- Entertainment Software Rating Board (ESRB) utilizado en Estados Unidos de América (Entertainment Software Association, 2017).

- Australian Classification Board (ACB) utilizadoen Australia (Australian Government - Department of Communications and the Arts, 2017).

- UnterhaltungssoftwareSelbstkontrolle (USK) utilizado en Alemania (Selbstkontrolle der Computerspielewirtschaft, 2017).

- Classificaçao Indicativa (ClassInd) utilizado en Brasil (Ministério da Justiça e Segurança Pública - Governo Federal, 2017)

- Infocomm Media Development Authority (IMDA) utilizadoenSingapur(Infocomm Media Development Authority - Singapore Government (IMDA), 2017).

- International Age Rating Coalition (IARC) es un unificador de sistemas de calificación utilizado para todo el mundo (International Age Rating Coalition (IARC), 2017) y están asociados todos los anteriores excepto IMDA. 
Tabla 1. Resumen de los sistemas de calificación de videojuegos (Fernández-Revelles et al., 2018).

\begin{tabular}{|c|c|c|c|}
\hline Sistema & Edades & Descriptores & Otros \\
\hline PEGI & $\begin{array}{l}\text { La clasificación por edades va encaminada a indi- } \\
\text { car la edad a la que serían idóneos los contenidos } \\
\text { sólo relacionados con la protección de menores y } \\
\text { sin entrar en el nivel de habilidad o dificultad. Los } \\
\text { niveles de clasificación son mayores de } 3,7,12,16 \\
\text { y } 18\end{array}$ & $\begin{array}{l}\text { Los descriptores son ocho e indican } \\
\text { los criterios por los cuales el juego ha } \\
\text { sido clasificado en una categoría deter- } \\
\text { minada. Las categorías son: Lenguaje } \\
\text { soez, Discriminación, Drogas, Miedo, } \\
\text { Juego, Sexo, Violencia, En línea }\end{array}$ & \\
\hline ESRB & $\begin{array}{l}\text { Niños Pequeños, Todos, Todos+10 es para } 10 \text { años } \\
\text { o más, Adolescentes para usuarios de } 13 \text { años } \\
\text { o más, Maduro para usuarios de } 17 \text { años o más, } \\
\text { Adultos únicamente sería solo para adultos de } 18 \\
\text { ańos o más, y Clasificación Pendiente }\end{array}$ & $\begin{array}{l}\text { Los descriptores de contenido indi- } \\
\text { can la motivación de la clasificación y } \\
\text { son: Referencia al alcohol, Animación } \\
\text { de sangre, Sangre, Derramamiento } \\
\text { de sangre, Violencia de caricatura, } \\
\text { Travesuras cómicas, Humor vulgar, } \\
\text { Referencia a drogas, Violencia de fan- } \\
\text { tasía, Violencia intensa, Lenguaje, Le- } \\
\text { tras de canciones, Humor para adultos, } \\
\text { Desnudez, Desnudez parcial, Apues- } \\
\text { tas reales, Contenido sexual, Temas } \\
\text { sexuales, Violencia sexual, Apuestas } \\
\text { simuladas, Lenguaje fuerte, Letra de } \\
\text { canción fuerte, Temas insinuantes, } \\
\text { Referencia al tabaco, Uso de alcohol, } \\
\text { Uso de drogas, Uso de tabaco, Violen- } \\
\text { cia, Referencias violentas }\end{array}$ & $\begin{array}{l}\text { Elementos interactivos informan } \\
\text { sobre la interactividad del pro- } \\
\text { ducto y son: Ubicación com- } \\
\text { partida, Interacción de usuarios, } \\
\text { Compras digitales, Internet sin } \\
\text { límites }\end{array}$ \\
\hline ACB & $\begin{array}{l}\text { La clasificación por edades y orientación de utili- } \\
\text { zación: CheckTheClassification (CTC) (verificar } \\
\text { clasificación) contenido evaluado y aprobado pero } \\
\text { falta la clasificación; General (G) es adecuada para } \\
\text { todos, tiene un impacto leve; Parental Guidance } \\
\text { (PG) (Orientación parental) no recomendado para } \\
\text { menores de } 15 \text { sin la orientación parental, tiene } \\
\text { impacto leve pero a veces puede ser confuso; Ma- } \\
\text { ture (M) (Maduro) recomendado para audiencias } \\
\text { maduras o adolescentes de más de } 15 \text { años por su } \\
\text { contenido con impacto moderado; MatureAccom- } \\
\text { panied (MA 15+) (Acompañado de adultos) re- } \\
\text { stringido y no adecuado para menores de } 15 \text { ańos, } \\
\text { los menores de } 15 \text { ańos deberían ir acompañados de } \\
\text { adultos por su fuerte impacto; Restricted (R 18+) } \\
\text { (Restringido) restringido a mayores de } 18 \text { ańos por } \\
\text { su contenido de alto impacto; Restricted (X 18+) } \\
\text { (Restringido) restringido a adultos mayores de } 18 \\
\text { años, especial restricción por contenido sexual ex- } \\
\text { plícito }\end{array}$ & $\begin{array}{l}\text { Los seis elementos clasificables sería } \\
\text { uno de los tres principios esenciales } \\
\text { de la decisión de clasificación. Los } \\
\text { seis elementos clasificables, como los } \\
\text { descriptores en otras categorías son: } \\
\text { Tema, Violencia, Sexo, Lenguaje, Uso } \\
\text { de drogas, Desnudos. }\end{array}$ & $\begin{array}{l}\text { Dos de los tres principios esen- } \\
\text { ciales de la decisión de la clasi- } \\
\text { ficación son: La importancia del } \\
\text { contexto, evaluación del impacto. }\end{array}$ \\
\hline USK & $\begin{array}{l}\text { Está realizada para la clasificación de los vide- } \\
\text { ojuegos. Utiliza una clasificación por edades: para } \\
\text { todos }(0) \text {, y mayores de } 6,12,16 \text { y } 18 \text { en las que } \\
\text { evalúa y califica que impacto puede tener ese juego } \\
\text { en función de diferentes aspectos. }\end{array}$ & $\begin{array}{l}\text { Estos descriptores o aspectos son: El } \\
\text { concepto de juego en función de su } \\
\text { realización audiovisual, Jugadas y } \\
\text { comprensión, Atmósfera, Realismo, } \\
\text { Autenticidad, Semejanza humana, } \\
\text { Atractivo para los jóvenes y potencial } \\
\text { identificación, Presión para actuar, } \\
\text { Violencia, Guerra, Miedo y amenaza, } \\
\text { Contenidos sexuales, Discriminación, } \\
\text { Lenguaje, Drogas }\end{array}$ & \\
\hline
\end{tabular}




\begin{tabular}{|c|c|c|c|}
\hline Sistema & Edades & Descriptores & Otros \\
\hline ClassInd & $\begin{array}{l}\text { Los niveles de clasificación por edad son: Libre } \\
\text { (L) para la audiencia general, (10) para mayores de } \\
10 \text { años, (12) para mayores de } 12 \text { años, (14) para } \\
\text { mayores de } 14 \text { años, (16) para mayores de } 16 \text { años, } \\
\text { (18) para mayores de } 18 \text { años. }\end{array}$ & $\begin{array}{l}\text { Los criterios o descriptores son tres: } \\
\text { violencia, sexo y drogas }\end{array}$ & $\begin{array}{l}\text { Los elementos que aumentan y } \\
\text { disminuyen el impacto de los cri- } \\
\text { terios son los atenuantes y agra- } \\
\text { vantes. Elementos atenuantes: } \\
\text { Composición de la escena, rel- } \\
\text { evancia, frecuencia, ligado a con- } \\
\text { tenidos: deportivos, históricos, } \\
\text { artísticos, culturales o científicos, } \\
\text { irónico o en contexto cómico- } \\
\text { lúdico, contexto de fantasía, } \\
\text { insinuación-simulación o prueba, } \\
\text { motivación, contrapunto, conte- } \\
\text { nido positivo. } \\
\text { Elementos agravantes: Com- } \\
\text { posición de la escena, relevancia, } \\
\text { frecuencia, valoración de conte- } \\
\text { nido negativo, motivación, con- } \\
\text { tenido inadecuado con nińos o } \\
\text { adolescentes y contexto }\end{array}$ \\
\hline
\end{tabular}

IMDA Singapur ve la necesidad de crear un sistema pro- Se basa en los principios generales pio de calificación de videojuegos para adaptarlo de: aceptación moral estándar genermejor a su cultura. En 2008 pone en marcha la alizada, necesidad de protección del calificación por edades que está estructurada así: menor, armonía religiosa y racial, inPara todas las edades $(\mathrm{G})$, para mayores de 6, 15,18 terés nacional, tratamiento del tema y y prohibido su uso y comercialización (X) o NAR contenido, evaluación del impacto y (NotAllowebforAll Rating) o no permitido para creatividad o mérito educativo. Utiliza todos los niveles. los siguientes descriptores: tema, violencia, sexo, desnudez, lenguaje, uso de drogas

\section{Discusión}

Creemos que las calificaciones de videojuegos juegan un papel muy importante en la protección de menores y adolescentes y en la orientación del uso adecuado de los videojuegos por esta razón vemos viable una calificación de juegos motores basada en los sistemas de calificación de videojuegos.

Para ello habrá conocer que estructura siguen los sistemas de calificación de videojuegos, extraer los patrones comunes en la calificación por edades y en los descriptores y añadir aquellas características que puedan aportar matices a la calificación aun no siendo comunes en todos los sistemas de calificación.

A partir de ahí tendremos que relacionar los descriptores de la calificación de los videojuegos con los descriptores y acciones que se pueden realizar en los juegos motores o que se realizan en las jugadas o "gameplay" de los juegos motores. El término gameplay es un término muy utilizado entre los jugadores de videojuegos.

La estructura que siguen los sistemas de calificación es similar en todos ellos. Utilizan unos escalones de edad donde se encuadrará el videojuego en función de la aparición o no de unas determinadas acciones relacionadas con los descrip- tores, o también puede determinar que se califique en diferente edad en función del impacto de esa acción puesto que puede verse influenciada la acción por potenciadores o inhibidores de ese impacto, a esos potenciadores o inhibidores del impacto los llamaremos moduladores.

Los moduladores del impacto serían los elementos del videojuego que intervienen en el videojuego de tal forma que en función de su interviención encontraríamos potenciadores e inhibidores de ese impacto.

Así en el sistema de calificación ClassInd(Ministério da Justiça e Segurança Pública - Governo Federal, 2017) aparecerían esos moduladores como agravantes o atenuantes, o en el sistema de calificación ACB (Australian Government - Department of Communications and the Arts, 2017) serían dos de los elementos a tener en cuenta para realizar la calificación y serían la importancia del contexto y la evaluación del impacto. $Y$ en el sistema de calificación ESRB serían ubicación compartida, interacción de usuarios, compras digitales, internet sin límites.

En los otros sistemas de calificación aparecen los moduladores integrados dentro de los descriptores como tales o con una subdivisión muy amplia de los descriptores.

Los moduladores los vamos a resumir en escenificación, 
contextualización, número de jugadores, interacción de jugadores, juego en línea y ganancias-premios o pérdidas-castigo.

Los descriptores aparecen muy diversificados en algunos casos por una matización muy específica que es muy útil, pero creemos que se podrían aglutinar los descriptores. Así se podría entender mejor como de unos descriptores muy genéricos con una influencia de los moduladores se modificaría el impacto del contenido.

Así todos los descriptores los podríamos englobar en tres descriptores principales violencia, sexo y adicciones. Dentro de estos descriptores caben todos encontrados en los sistemas de calificación de videojuego y tendrían mayor o menor impacto en función de la actuación de los moduladores.

Puede que a simple vista parezca que algunos descriptores encontrados en los sistemas de calificación de videojuegos encontrados no estén como pueden ser: lenguaje soez, miedo, desnudos, drogas, juegos de apuestas ahora explicamos donde se encuadran.

Lenguaje soez por ejemplo que aparece en ClassInd(Ministério da Justiça e Segurança Pública - Governo Federal, 2017) se podría incluir dentro del descriptor violencia, como violencia en el lenguaje.

Miedo que aparece en PEGI (Pan European Game Information (PEGI), 2017) se podría incluir dentro del descriptor violencia unido a los moduladores de contextualización y escenificación.

En el descriptor sexo se incluirían todas las acciones con referencia a este aunque sean simples desnudos como aparecen en la calificación USK (Selbstkontrolle der Computerspielewirtschaft, 2017).

Drogas, alcohol, tabaco, consumo de sustancias prohibidas, juegos de azar y apuestas que aparecen por ejemplo en ESRB y PEGI (Entertainment Software Association, 2017; Pan European Game Information (PEGI), 2017) se incluirían dentro del descriptor de adicciones.

Dentro de las adicciones en los juegos de azar y apuestas se modificaría su impacto en función de los moduladores ganancias-premios o pérdidas castigo.

Ya con estos descriptores junto a sus moduladores podríamos hacer una unificación de las escalas de calificación por edades, puesto que en los rangos de edad hay una gran coincidencia en todos los sistemas de calificación de videojuegos. La calificación unificada de los sistemas de calificación de videojuegos sería:

- Pendiente de calificación: Cuando está evaluándose y falta algún trámite burocrático como ocurre con la calificación CTC que realiza ACB (Australian Government - Department of Communications and the Arts, 2017).

- General, para todos los públicos o también podría llamarse para mayores de 3 ańos puesto que antes no tendrían conciencia de lo que ven. En el que podrían in- cluirse: acciones relacionadas con los tres descriptores, pero muy escenificadas y contextualizadas inhibiendo el impacto, y tendrían impacto leve como podría ocurrir con dibujos animados.

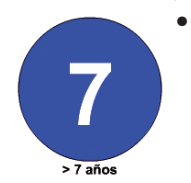

- Mayores de 7: cuando existe algo de violencia, violencia con miedo, pero está escenificado y contextualizado inhibiendo el impacto para no ser explicito, por lo cual también podrían participar los 3 descriptores.

- Mayores de 12 años: en esta categoría también podrían estar los 3 descriptores y podría aparecer violencia no llegando a ser totalmente explicita, acciones con violencia en el lenguaje, acciones en las que se hace referencia a los juegos de azar y apuestas que estarían en las adicciones. Igualmente aparecerían desnudos y referencias al sexo pero no explícitos.

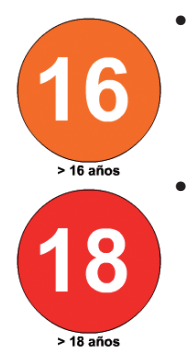

- Mayores de 16 años: Aquí podrían encontrarse los 3 descriptores casi totalmente explícitos incluso con moduladores potenciadores.

- Mayores de 18 ańos: Se podrían encontrar todos los descriptores potenciados al máximo por los modulares, sólo teníendo un límite el de las leyes de ese determinado país como ocurre con IMDA en el que está prohibida la comercialización de videojuegos en los que por ejemplo aparezca violencia sexual (Infocomm Media Development Authority - Singapore Government (IMDA), 2017).

- Prohibidos: Aquellos videojuegos en los que por lo explícito del contenido han llegado a ser prohibidos.

Como se aprecia se ha escogido la escala de edades de PEGI (Pan European Game Information (PEGI), 2017) con algunas matizaciones.

Ahora pasamos a trasladar este sistema unificado de calificación de videojuegos y tratamos de adaptarlo a los juegos motores.

\section{Conclusiones}

Se pueden establecer las bases para una propuesta de un sistema de calificación, evaluación y clasificación de juegos motores partiendo de los sistemas de calificación de videojuegos con algunas modificaciones como indicamos en los siguientes párrafos. 
Bases para una propuesta de sistema de calificación de Juegos Motores.

En primer lugar, seria observar que en los juegos motores participan e interactúan personas vivas con objetos reales con los que se pueden producir golpes, lesiones, etc..., aunque en el juego motor se esté vivenciando una ficción. Por eso cuando hay muertes en el juego se referirá a la vida en la ficción, no a muerte real. Sin embargo, cuando se hable de golpeos y peleas hay que aclarar si con en la ficción y atenudas con elementos del juego y son reales.

En segundo lugar, la calificación de los juegos motores ha de ser válida tanto para la propuesta de un juego motor escrito, planificado que lo leemos en un libro o blog de juegos. En este caso hay que hacer un esfuerzo de abstracción para adivinar qué tipo de acciones se puden dar en el juego motor. También ha de ser válida para grabaciones de juegos motores, o jugadas o "gameplay" de un determinado juego motor, puesto que nunca un juego motor es jugado igual en diferentes ocasiones como vemos en este ejemplo de 6 jugadas diferentes del mismo juego motor par-impar (Fernández-Revelles, 2017a, 2017b, 2017c, 2017d, 2017e, 2017f).

En tercer lugar hay que tener en cuenta que los sistemas de calificación deben de sugerir edades de uso adecuado y además orientar en el uso (Laczniak et al., 2017), por lo tanto no se puede pasar por alto que en los juegos motores existe un contenido principal que es el contenido motor que hay que incluirlo como descriptor en función de la dificultad, intensidad, etc..

En nuestro sistema de calificación de juegos motores igualmente contaríamos con: moduladores, descriptores y la escala de clasificación por edades.

La calificación por edades por edades podría ser similar a la unificada que hemos descrito anteriormente, aunque habría que incluir además la categoría de si es adecuada para mayores de 65 años.

Los descriptores igualmente contaríamos con violencia, sexo y adicciones a los que habría que ańadir los referentes al ejercicio físico y actividad motora.

En cuanto a los moduladores se podrían contar con los mismos que en la calificación unificada descrita anteriormente a los que habría que añadir los que pudiesen afectar a la actividad motora.

\section{Limitaciones del estudio y perspectivas de futuro}

Para proponer un sistema de calificación de los juegos motores tendríamos analizarlo a través de un grupo de expertos para concretar las calificaciones por edad, descriptores y moduladores. Al mismo tiempo configurar un cuestionario en el que la calificación por edades esté claramente especificada por los descriptores y moduladores de estos. Ya confeccionado el cuestionario habría que realizar un análisis factorial exploratorio y otro confirmatorio para validar el cuestionario.

\section{Referencias}

1. Anders, K. (1999). Marketing and policy considerations for violent video games. Journal of Public Policy \& Marketing, 18(2), 270-273.

2. Australian Government - Department of Communications and the Arts. (2017). Australian Classification Board (ACB). Retrieved 1/09/2017, from http://www.classification.gov.au/Pages/Home.aspx

3. Becker-Olsen, K. L., \& Norberg, P. A. (2010). Caution, Animated Violence Assessing the Efficacy of Violent Video Game Ratings. Journal of Advertising, 39(4), 83-94. doi: 10.2753/joa0091-3367390406

4. Bijvank, M. N., Konijn, E. A., Bushman, B. J., \& Roelofsma, P. (2009). Age and Violent-Content Labels Make Video Games Forbidden Fruits for Youth. Pediatrics, 123(3), 870-876. doi: 10.1542/peds.2008-0601

5. Cranwell, J., Whittamore, K., Britton, J., \& Leonardi-Bee, J. (2016). Alcohol and Tobacco Content in UK Video Games and Their Association with Alcohol and Tobacco Use Among Young People. Cyberpsychology Behavior and Social Networking, 19(7), 426-434. doi: 10.1089/ cyber.2016.0093

6. Dogruel, L., \& Joeckel, S. (2013). Video game rating systems in the US and Europe: Comparing their outcomes. International Communication Gazette, 75(7), 672-692. doi: 10.1177/1748048513482539

7. Entertainment Software Association. (2017). Entertainment Software Rating Board (ESRB). Retrieved 1/09/2017, from http://www.esrb.org

8. Fernández-Revelles, A. B. (2012). ABFR-Index: correlación entre producción científica y Juegos Olímpicos 2008. Habilidad Motriz, 38, 5157. doi: http://hdl.handle.net/10481/29520

9. Fernández-Revelles, A. B. (2014). ABFR-index: correlación entre producción científica en "fútbol" y ranking / ABFR-index: correlation between "soccer" scientific production and ranking. Revista Internacional de Medicina y Ciencias de la Actividad Física y el Deporte, 14(56), 705718.

10. Fernández-Revelles, A. B. (Producer). (2017a, Oct 04, 2017). Juego Parimpar Fase 0 - 5R - 00207. Juegos Motores. [Archivo de vídeo] Retrieved from https://youtu.be/BhKf-8J1fg4

11. Fernández-Revelles, A. B. (2017b). Juegos Motores (Blog - Blogger). Retrieved 1/09/2017, from http://juegosmotores2.blogspot.com.es/

12. Fernández-Revelles, A. B. (2017c). Juegos Motores (Blog - Tumblr). Retrieved 1/09/2017, from https://www.pinterest.es/juegosmotores/

13. Fernández-Revelles, A. B. (2017d). Juegos Motores (Instagram). Retrieved 1/09/2017, from https://www.instagram.com/juegos_motores/

14. Fernández-Revelles, A. B. (2017e). Juegos Motores (Pinterest). Retrieved 1/09/2017, from https://juegosmotores.tumblr.com/

15. Fernández-Revelles, A. B. (2017f). Juegos Motores (Twitter). Retrieved 1/09/2017, from https://twitter.com/juegosmotores

16. Fernández-Revelles, A. B. (2017g). Juegos Motores (Youtube). Retrieved 1/09/2017, from https://www.youtube.com/user/juegosmotores

17. Fernández-Revelles, A. B., Chillon, P., Martinez, J. M., Huertas, F. J., Robles, A., Dafos, J., . . Delgado, M. (2009). Evaluation of research on fibromialgia in Spain. Gaceta sanitaria, 23, 214-214.

18. Fernández-Revelles, A. B., Robles, A., Dafos, J., Soto, V. M., Perez-Cortes, A. J., Latorre, P., . . Romero, C. (2009). Physical activity: Evaluation of research in Spain. Gaceta sanitaria, 23, 204-204.

19. Fernández-Revelles, A. B., Zurita-Ortega, F., Castañeda-Vázquez, C., Martínez-Martínez, A., Padial-Ruz, R., \& Chacón-Cuberos, R. (2018). Sistemas de calificación de video juegos, revisión narrativa. ESHPA Education, Sport, Health and Physical Activity, 2(1), 62-74. 
20. Funk, J. B., Flores, G., Buchman, D. D., \& Germann, J. N. (1999). Rating electronic games - Violence is in the eye of the beholder. Youth \& Society, 30(3), 283-312. doi: 10.1177/0044118x99030003002

21. Gentile, D. A., Maier, J. A., Hasson, M. R., \& de Bonetti, B. L. (2011) Parents' Evaluation of Media Ratings a Decade After the Television Ratings Were Introduced. Pediatrics, 128(1), 36-44. doi: 10.1542/ peds.2010-3026

22. Infocomm Media Development Authority - Singapore Government (IMDA). (2017). Infocomm Media Development Authority (IMDA), Retrieved 1/09/2017, from https://www.imda.gov.sg/

23. International Age Rating Coalition (IARC). (2017). International Age Rating Coalition (IARC). Retrieved 1/09/2017, from https://www. globalratings.com/

24. Jordan, A. B. (2008). Children's media policy. Future of Children, 18(1), 235-253.

25. Laczniak, R. N., Carlson, L., Walker, D., \& Brocato, E. D. (2017). Parental Restrictive Mediation and Children's Violent Video Game Play: The Effectiveness of the Entertainment Software Rating Board (ESRB)
Rating System. Journal of Public Policy \& Marketing, 36(1), 70-78. doi: 10.1509/jppm.15.071

26. Ministério da Justiça e Segurança Pública - Governo Federal. (2017). Classificaçao Indicativa (ClassInd). Retrieved 1/09/2017, from http:// www.justica.gov.br/seus-direitos/classificacao

27. Pan European Game Information (PEGI). (2017). Pan European Game Information (PEGI). Retrieved 1/09/2017, from http://www.pegi.info/ es/

28. Sedeno, A. (2010). Videogames as cultural devices: development of spatial skills and application in learning. Comunicar(34), 183-189. doi: 10.3916/c34-2010-03-018

29. Selbstkontrolle der Computerspielewirtschaft. (2017). Unterhaltungssoftware Selbstkontrolle (USK). Retrieved 1/09/2017, from http://www.usk.de/

30. Walsh, D. A., \& Gentile, D. A. (2001). A validity test of movie, television, and video-game ratings. Pediatrics, 107(6), 1302-1308. doi: 10.1542/peds.107.6.1302 